\title{
p63+ ureteric bud tip cells are progenitors of intercalated cells
}

\author{
Samir S. El-Dahr, ${ }^{1}$ Yuwen Li, Jiao Liu, ${ }^{1}$ Elleny Gutierrez, ${ }^{1}$ Kathleen S. Hering-Smith, ${ }^{2}$ \\ Sabina Signoretti, ${ }^{3}$ Jean-Christophe Pignon, ${ }^{3}$ Satrajit Sinha, ${ }^{4}$ and Zubaida Saifudeen ${ }^{1}$ \\ 'Department of Pediatrics and 'Department of Physiology, Tulane University School of Medicine, New Orleans, Louisiana, \\ USA. ${ }^{3}$ Department of Pathology, Brigham and Women's Hospital, Harvard School of Medicine, Boston, Massachusetts, \\ USA. ${ }^{4}$ Department of Biochemistry, Jacobs School of Medicine, University of Buffalo, New York, USA.
}

\begin{abstract}
During renal branching morphogenesis, ureteric bud tip cells (UBTC) serve as the progenitor epithelium for all cell types of the collecting duct. While the transcriptional circuitry of ureteric bud (UB) branching has been intensively studied, the transcriptional control of UBTC differentiation has been difficult to ascertain. This is partly due to limited knowledge of UBTCspecific transcription factors that mark the progenitor state. Here, we identify the transcription factor p63 (also known as TP63), a master regulator of basal stem cells in stratified epithelia, as a specific marker of mouse and human UBTC. Nuclear p63 marks Ret ${ }^{+}$UBTC transiently and is silenced by the end of nephrogenesis. Lineage tracing revealed that a subset of UBTC expressing the $\Delta \mathrm{Np} 63$ isoform ( $\mathrm{N}$-terminus truncated $\mathrm{p63}$ ) is dedicated to generating cortical intercalated cells. Germline targeting of $\triangle \mathrm{Np} 63$ in mice caused a marked reduction in intercalated cells near the time of birth, indicating that p63 not only marks UBTC, but also is essential for their differentiation. We conclude that the choice of UBTC progenitors to differentiate is determined earlier than previously recognized and that UBTC progenitors are prepatterned and fate restricted. These findings prompt the rethinking of current paradigms of collecting duct differentiation and may have implications for regenerative renal medicine.
\end{abstract}

Conflict of interest: The authors have declared that no conflict of interest exists.

Submitted: August 23, 2016

Accepted: March 23, 2017

Published: May 4, 2017

Reference information: JCl Insight. 2017;2(9):e89996. https:// doi.org/10.1172/jici.insight.89996.

\section{Introduction}

The developing kidney is derived from the caudal intermediate mesoderm by iterative mesenchymal-epithelial interactions between the metanephric mesenchyme and ureteric bud (UB) cell lineages. The collecting ducts, which are derivatives of the UB, play a critical role in the maintenance of salt, water, and acid-base homeostasis. These physiological functions are carried out by highly specialized cell types: the principal cells (PC) and intercalated cells (IC). Embryologically, PC and IC are believed to be derived from bipotent UB precursors, which are themselves derived from stem/progenitor cells located at the UB tips (1). Insults that disrupt the ureteric bud tip cell's (UBTC's) capacity for cell renewal and/or terminal differentiation have the potential to cause structural defects and dysregulation of blood pressure, electrolyte and water balance, and acid-base homeostasis $(2,3)$. Designing strategies to hasten collecting duct regeneration following injury and rebuilding a functional collecting duct from pluripotent cells will benefit from a better understanding of UBTC fate determinants.

While much is known about the cellular and molecular mechanisms governing UB growth and branching, relatively little is known about the dynamic regulation of UB cell differentiation. Careful lineage analysis has established that UBTC are the progenitors of the entire collecting duct epithelium (4-6). In response to glial-derived neurotrophic factor released from the surrounding mesenchyme, Ret $^{+}$UBTC undergo proliferation, changes in cell shape, and cell movements (collectively, a process called mitosis-associated cell dispersal) to mediate UB growth and branching and, ultimately, form the renal collecting system (5). There is limited knowledge of the UBTC transcriptome at the earliest stages of mouse and rat kidney development (7). Current models posit that UBTC give rise to bipotent UB precursors capable of differentiating to PC or IC and that Notch and Foxi1 signaling dictate the decisions for fate commitment and/or are required for fate maintenance $(2,8,9)$. However, a number of fundamental questions remain unanswered. For example, can UBTC differentiate directly to PC or IC without passing through the bipotent stage? Where does the commitment step take place (UB tip vs. trunk)? And, what are the transcription factors that specify the cell type fates? 
p63 (also known as TP63) is a member of the p53 gene family of transcription factors (which comprises $\mathrm{p} 53$, p63, and $\mathrm{p} 73$ ). The $\mathrm{p} 63$ gene is transcribed into full-length (TAp63) and N-terminus-truncated $(\Delta \mathrm{Np} 63)$ transcripts via differential promoter utilization. Differential splicing of $p 63$ also generates multiple C-terminus isoforms, the exact functions of which are largely unknown (10-14). p63 is expressed in the basal layer of stratified epithelia such as the skin, lung, and limbic epithelium. Genetic studies have shown that p63 is absolutely required for epithelial tissue morphogenesis (15-19), as mice lacking p63 isoforms die in the perinatal period due to severe skin disease and dehydration. Emerging data also implicate p63 in the regeneration program of the alveolar epithelium following injury (20). As stated earlier, traditionally, p63 expression is observed in basal epithelial stem cells of stratified epithelia. Here, we show for the first time to our knowledge that p63 is expressed in the UB tip domain, which consists of a simple progenitor epithelium. p63 expression in the UB tip is transient and is silenced by the end of nephrogenesis. Moreover, a subset of UBTC expressing the $\triangle \mathrm{Np} 63$ isoform gives rise to cortical IC. Finally, gene targeting in mice directly implicates $\Delta \mathrm{Np} 63$ in IC differentiation. Thus, in sharp contrast to existing models of collecting duct differentiation, our findings suggest that UB tip progenitors are prepatterned and fate restricted.

\section{Results}

p63 transiently marks UBTC in mouse and human kidneys. Although p63 expression in the urothelium is well described, to our knowledge, its expression in the nephron epithelium has not been reported previously. To characterize the spatiotemporal expression of p63 during kidney development, we immunostained sections of embryonic (E12.0-E17.5) and postnatal kidneys (P0, P3, P5, P10, and P60) with monoclonal p63 antibodies (clones 4A4 and SFI-6), which recognize both $\triangle \mathrm{Np63}$ and TAp63 isoforms. At E12.0, the UB had branched 2-3 times; both UB and its branches were p63 negative (Figure 1A). p63 expression in the UB epithelium was first detectable at E13.5, but at this stage, p63 was not detectable in the UB branches or tips (Figure 1B). E15.5 marked the onset of p63 expression in UBTC (Figure 1, C and D). At E17.5, p63 was abundantly expressed in UBTC, and it remained so until it was permanently silenced by P5 (Figure 1, E-I). After P5, p63 immunoreactivity was limited to the pelvic urothelium (Figure $1 \mathrm{~J}$ ). Collectively, these findings demonstrate that p63 is a marker of UBTC and is expressed only transiently during kidney development.

The c-Ret tyrosine kinase receptor marks the UB tip after E12.5, and Ret signaling is absolutely required for UB outgrowth and branching (21). We asked if p63 expression overlaps with that of Ret. For this purpose, we used Ret-EGFP knockin mice (22). In these mice, EGFP expression is regulated by the Ret endogenous regulatory elements. We observed that $\mathrm{p} 63$ labels a subset of $\mathrm{GFP}^{+}$UBTC (Figure 2). The distribution of $\mathrm{p}^{+} 3^{+}$cells within the UB tip domain was not spatially associated with branching points.

We next asked if p63 expression in the UB tip, observed in the mouse kidney, is conserved in human kidneys. Immunostaining of a fetal human kidney at 12 weeks after conception revealed that p63 was expressed in the main UB branches (Figure 3, A-D) and UB tips (Figure 3E). Thus, expression of p63 in UBTC is evolutionarily conserved and, therefore, is likely to have functional importance.

$\triangle N p 63^{+}$UBTC give rise to cortical IC. Previous studies have shown that $\triangle \mathrm{Np} 63$ labels keratinocyte, lung, and prostate stem cells and is required for their maintenance and differentiation (16, 19, 20, 23-25). We asked whether $\Delta \mathrm{Np} 63$ serves a similar function in the UB progenitors. To this end, we first defined the spatial expression of $\Delta \mathrm{Np} 63$ in the UB tip. Using $\Delta \mathrm{Np} 63$ isoform-specific antibody (26), we found 1-2 cells per tip that stained positive for $\Delta \mathrm{Np} 63$ (Figure 4, A-D). We also examined knockin $\Delta \mathrm{Np} 63^{+/ \text {gfp }}$ mice (which express GFP under the control of the endogenous $\triangle \mathrm{Np} 63$ regulatory elements) (26). We clearly identified $\mathrm{GFP}^{+} \mathrm{UB}$ tips/cells in whole mount kidneys (Figure 4E) and tissue sections (Figure 4F), respectively. Costaining of GFP with p63 and Pax2 revealed that $\triangle$ Np63-expressing cells constituted a subset of total p63 ${ }^{+}$tip cells, whereas the cap mesenchyme was devoid of p63 (Figure 4, G-I).

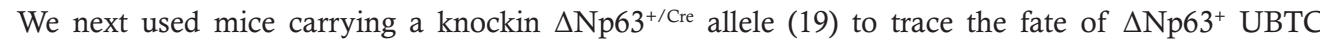
$\left(\mathrm{UBTC}^{\triangle \mathrm{Np} 63}\right.$ ). We performed single- or double-label immunostaining in fetal and neonatal kidney sections from $\Delta \mathrm{Np} 63^{+/ \mathrm{Cre}} ; \mathrm{R} 26 \mathrm{R}^{\mathrm{EYFP}}$ or $\mathrm{R} 26 \mathrm{R}^{\mathrm{mT} / \mathrm{mG}}$ mice. At E17.5-E18.5, we documented the presence of 1-3 $\mathrm{EYFP}^{+} / \mathrm{p} 63^{+}$cells per UB tip domain (Figure 5), indicating that we are likely detecting the earliest $\Delta$ Np63-expressing cells. As expected, the pelvic urothelium contained $\mathrm{p}^{+} 3^{+} / \mathrm{EYFP}^{+}$and daughter $\mathrm{p} 63^{-} /$ $\mathrm{EYFP}^{+} \Delta \mathrm{Np} 63^{\text {lineage }}$ cells (Figure $5 \mathrm{~A}$ ). By P1, labeled $\Delta \mathrm{Np} 63^{\text {lineage }}$ cells were detectable not only in the UB tip, but also in the collecting ducts (Figure 6, A-D). While we found labeled $\Delta \mathrm{Np} 63^{\text {lineage }}$ cells in the pelvic epithelium, we did not observe any labeled cells in the papillary collecting ducts, making it highly unlikely that labeled cells found in the cortical collecting ducts migrated up from the pelvic epithelium (Figure 6E). 

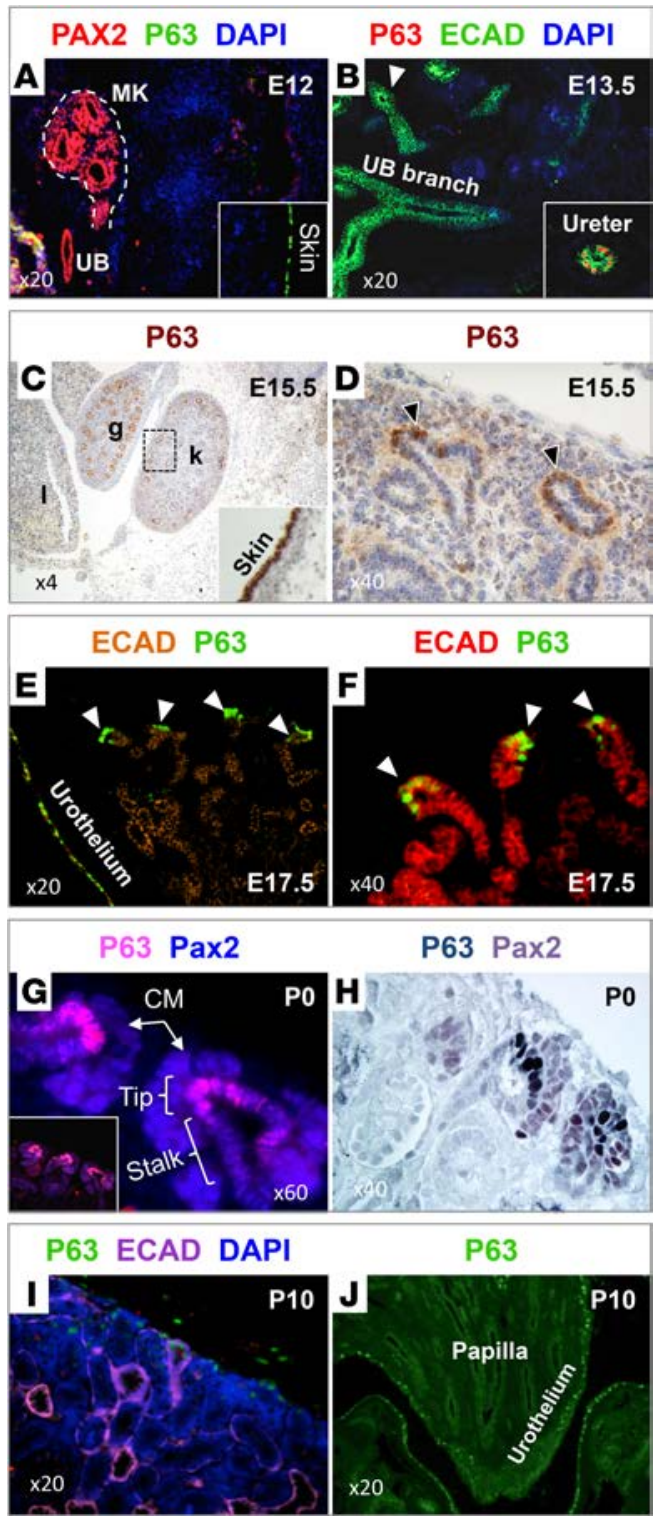

od but die postnatally of severe skin disease (26), precluding the ability to examine the adult kidney phenotype. However, histological and immunohistochemical analysis of late-gestation $\Delta \mathrm{Np} 63^{\mathrm{gfp} / \mathrm{gfp}}$ mice revealed that heterozygous or homozygous deletion of $\Delta N p 63$ has no discernible effect on UB integrity or branching (Figure 9, A and B, and data not shown). The residual p63 immunoreactivity in $\Delta \mathrm{Np} 63^{\text {gp/gfp }}$ kidneys (Figure 9, C and D) likely reflects the TAp63 isoform, which also localizes to the UB tip (Saifudeen and Liu, unpublished observations). Importantly, although loss of $\Delta \mathrm{Np} 63$ did not alter the integrity of $\mathrm{GFP}^{+}$tip progenitors (Figure 9, C and D, insets), it caused a remarkable reduction (but not complete elimination) in abundance of cortical CAII ${ }^{+} \mathrm{IC}$ (Figure 9, E and F). Thus, $\Delta \mathrm{Np} 63$ is required for the maintenance (renewal, survival, or differentiation) of a subset of UB tip progenitors that are able to transition into IC. The precise mechanism(s) will need to be addressed in future studies.

\section{Discussion}

The present study demonstrates that the transcriptional regulator, p63, is a specific marker of Ret $^{+}$UBTC, which are the progenitors of all cell types of the mature collecting duct. Interestingly, expression of p63 in the UB tip is transient, beginning around E15 and ending at P5, thus coinciding temporally with the initiation of specification and terminal differentiation of the collecting duct. Fate tracing of UBTC expressing the $\Delta \mathrm{Np} 63$ isoform revealed that $\mathrm{UBTC}^{\Delta \mathrm{Np} 63 \text { lineage }}$ give rise to cortical IC. Genetic deletion of $\triangle N p 63$ interferes with UBTC differentiation into cortical IC. Collectively, these findings have uncovered 


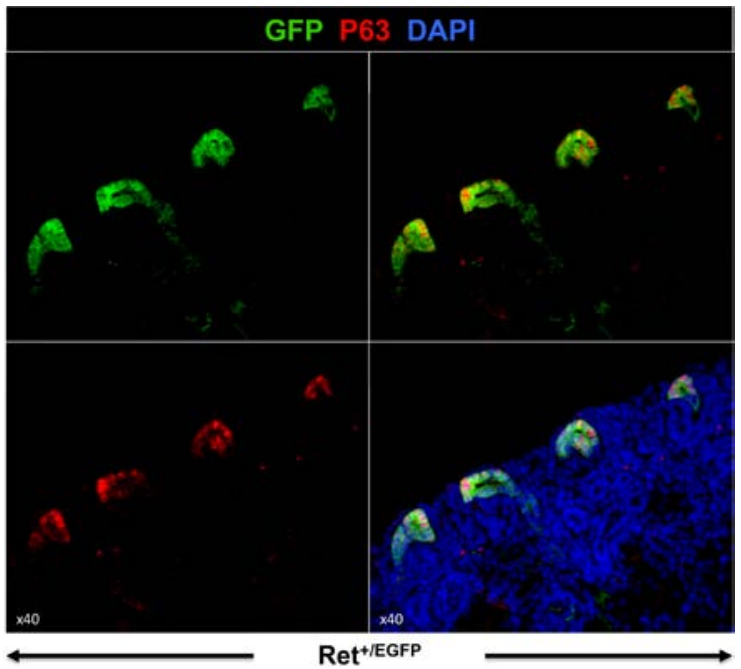

Figure 2. p63 labels a subset of Ret $^{+}$ureteric bud tip cells. Section immunofluorescence in P1 kidneys of Ret ${ }^{+/ E C F P}$ mice. Ret/EGFP (green) marks the entire ureteric bud (UB) tip domain, whereas p63 marks a subset of EGFP+ cells. The distribution of $p 63^{+}$cells in the UB tip is not associated with branching points $(n=3)$. Original magnification, $\times 40$.

a transcriptional regulator of UBTC differentiation and demonstrate that at least a subset of UBTC progenitors are sorted out early and differentiate into IC.

During kidney development, as the UB branches and elongates, the tip and trunk domains are patterned into progenitors and progeny, respectively. This tip-to-trunk program is reflected by differences in gene expression (7). While it is recognized that UBTC are the progenitors of all collecting duct cell types (29), the mechanisms that balance their renewal and differentiation are not well defined. Local Wnt- $\beta$-catenin signaling may prevent premature differentiation of UBTC, probably acting via Sox9 and Emx2 $(30,31)$. Extrinsic factors released from the cap mesenchyme, stroma, or neighboring epithelial elements may also impinge on UBTC differentiation. Regardless of the initial differentiation signal, current models posit that UBTC progenitors differentiate into bipotential UB precursors $(1,32)$, and that acquisition of Foxi1 activity in these precursors jump starts the IC differentiation program (32), as $\mathrm{Foxi1}^{-1-}$ mice develop distal renal tubular acidosis due to lack of IC (33). In contrast, Notch signaling is necessary to initiate and maintain the PC fate and, possibly, to suppress Foxil signaling. This was illustrated in mice lacking mindbomb-1 (mib-1), an E3 ubiquitin ligase that mediates ligand processing in the Notch-releasing cell. Mib-1-deficient kidneys have a markedly reduced PC/IC ratio, although the overall PC and IC patterning in the collecting duct remained unaltered (34). An untested hypothesis for UBTC differentiation is that a small subset of tip cells are sorted out ear$1 y$ in the process for either PC or IC fate under the influence of a specific developmental regulator. Our findings lend support to this hypothesis. In the present work, we report that UBTC ${ }^{\triangle \mathrm{Np} 63 \text { lineage }}$ consistently give rise to cortical IC (both $\mathrm{B}$ type and A type). Although occasional UBTC ${ }^{\Delta \mathrm{Np} 63 \text { lineage }}$ labeled cells were found in the medullary collecting ducts, these cells lacked vB1-ATPase expression. Therefore, our findings support the idea that cortical type B IC give rise to type A IC, previously proposed by Al-Awqati (reviewed in ref. 2). The cellular precursor of medullary type A IC remains to be determined.

The present study provides several lines of evidence supporting the idea that $\Delta \mathrm{Np} 63$ participates

Figure 3. p63 is expressed in ureteric bud tip cells of human fetal kidneys. A tissue section from a 12-week-old human fetus stained with p63 (4A4) antibody. (A and B) Low- and high-power images of the central ureteric bud (UB) branches, which express p63. (C-E) p63 is expressed in cortical collecting ducts and in the tip domain ( $n=1$ fetal kidney). Original magnification, $\times 10$ (A and C); $\times 40$ (D and E); $\times 60$ (B).

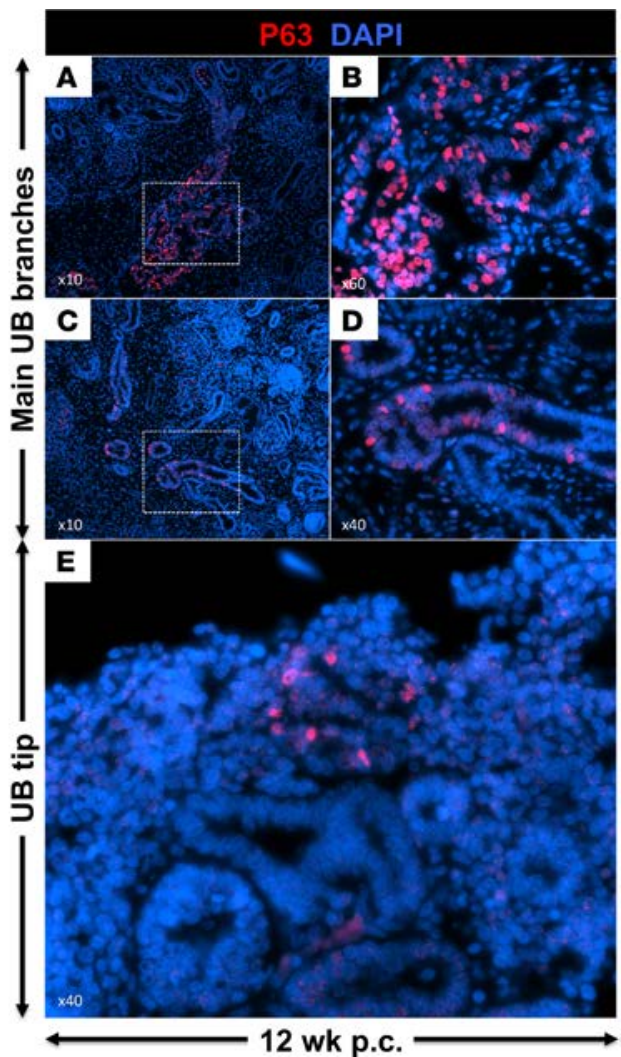




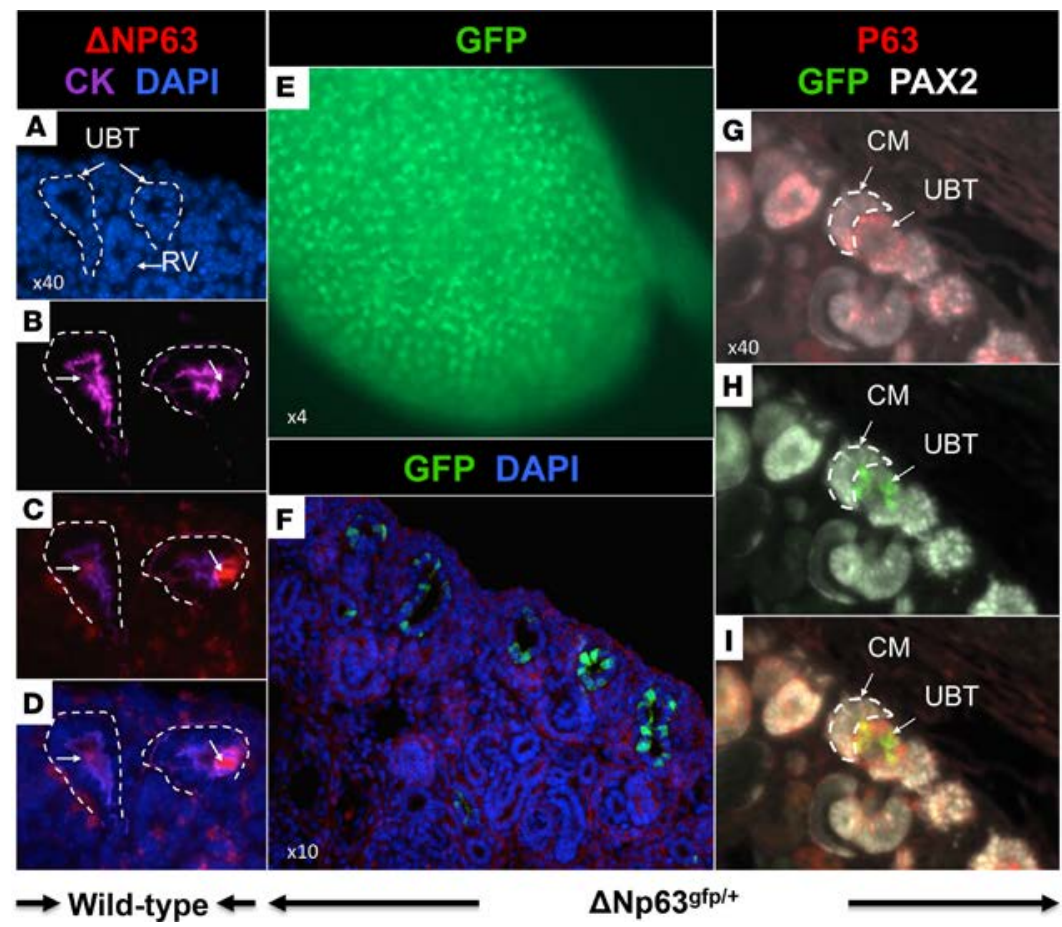

Figure 4. $\triangle \mathrm{Np63}$ is expressed in a subset of ureteric bud tip cells. (A-D) Section immunofluorescence of an E17.5 kidney using $\Delta$ Np63-specific antibody. Staining for cytokeratin (CK) identifies the ureteric bud (UB) branches. Arrows point to $\triangle \mathrm{Np63}^{+}$cells in B-D. RV, renal vesicle. (E) Whole mount GFP fluorescence in an E18.5 kidney from a $\Delta \mathrm{Np} 3^{+/ g f p}$ knockin mouse. Note the tip expression of GFP. (F) Section immunofluorescence using

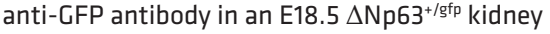
showing UB tip-specific expression of GFP. (G-I) Section immunofluorescence using anti-GFP, p63,

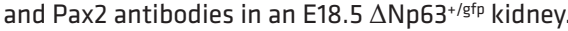
p63 (red) labels the UB tip domain (UBT). Pax2 (gray) labels the UB tip (UBT) and surrounding cap mesenchyme (CM). GFP (green), representing $\triangle \mathrm{Np63}$, is expressed in a subset of $p 63^{+}$ureteric bud tip cells ( $n=2-5$ animals/experiment). Original magnification, $\times 4(\mathbf{E}) ; \times 10(\mathbf{F}) ; \times 40(\mathbf{A}-\mathbf{D}$ and $\mathbf{G}-\mathbf{I}$ ).

in programming UBTC-to-IC fate. (a) $\triangle \mathrm{Np} 63$ is expressed at the right location (UB tip) and time (late gestation), which coincides with collecting duct cell functional specification. (b) $\Delta \mathrm{Np} 63$ acts transiently, as it is completely silenced soon after birth. This finding suggests that $\triangle \mathrm{Np} 63$ initiates the UBTCto-IC program, but its presence is not essential to maintain the differentiation state; another factor such as Foxi1 may play such a role. Indeed, loss of Foxi1 is accompanied by accumulation of precursor cells expressing both PC and IC markers (33), confirming that Foxi1 is necessary for the later stages of IC specification. (c) Genetic deletion of $\triangle N p 63$ results in a reduction of the cortical IC mass. Unfortunately, $\Delta \mathrm{Np} 63^{-/-}$mice die shortly after birth, precluding the full characterization of the mature phenotype and physiological effect on the urinary acidification mechanism. Our attempts at sampling the amniotic fluid of late-gestation $\Delta \mathrm{Np} 63^{-/-}$mice failed due to the contamination of the fluid with debris from sloughed skin and epidermal tissues due to extreme fragility of the skin and the amniotic sac. Although this issue cannot be addressed at the present time, based on the findings in the present study, we predict that UB-specific deficiency of $\Delta \mathrm{Np} 63$ will lead to permanent loss of

Figure 5. Fate tracing of UBTC ${ }^{\triangle N p 63}$ in E17.5 $\triangle$ Np63 $^{\text {+/Cre; ROSA26 EVFP }}$ mice. (A and B) Section immunohistochemistry. p63 (brown) strongly labels the ureteric bud tip cells (UBTC) and pelvic urothelium. EYFP (purple) labels 1-2 UBTC (white arrowheads) and patches of pelvic epithelial cells (black arrowheads). (C and D) Colocalization of p63 protein (purple) with EYFP (green) in UBTC ( $n=$ 5). Arrowheads point to ${\mathrm{p} 63^{+} / \mathrm{EYFP}}^{+}$cells. Original magnification, $\times 10(\mathbf{A}) ; \times 20(B) ; \times 40(C$ and D) .

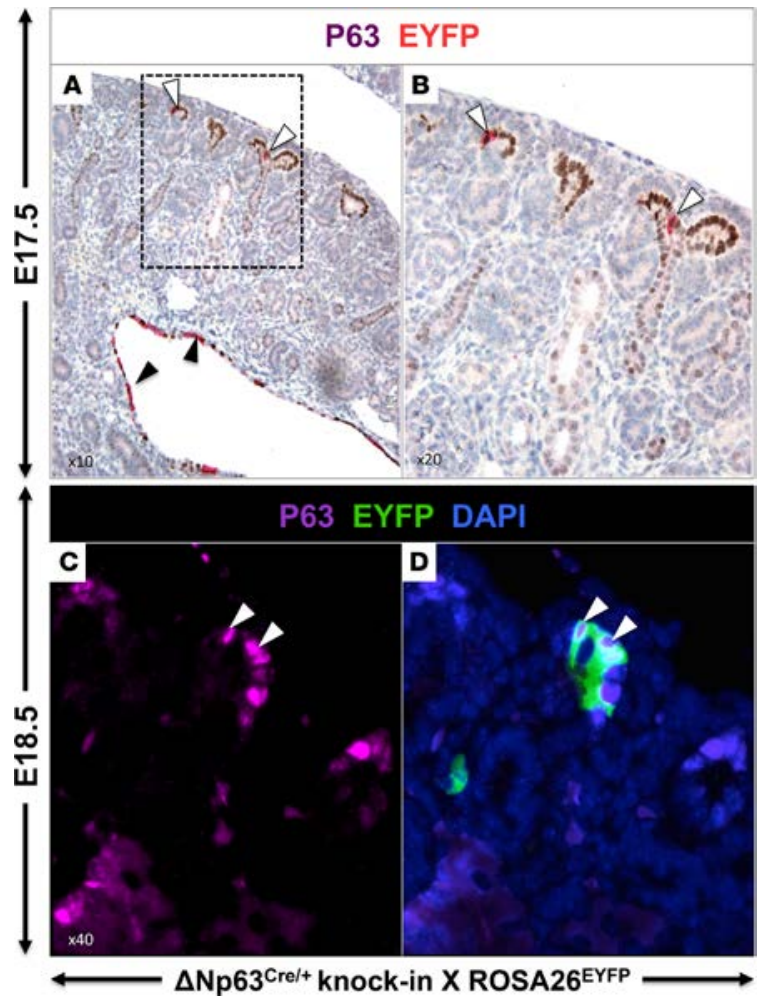




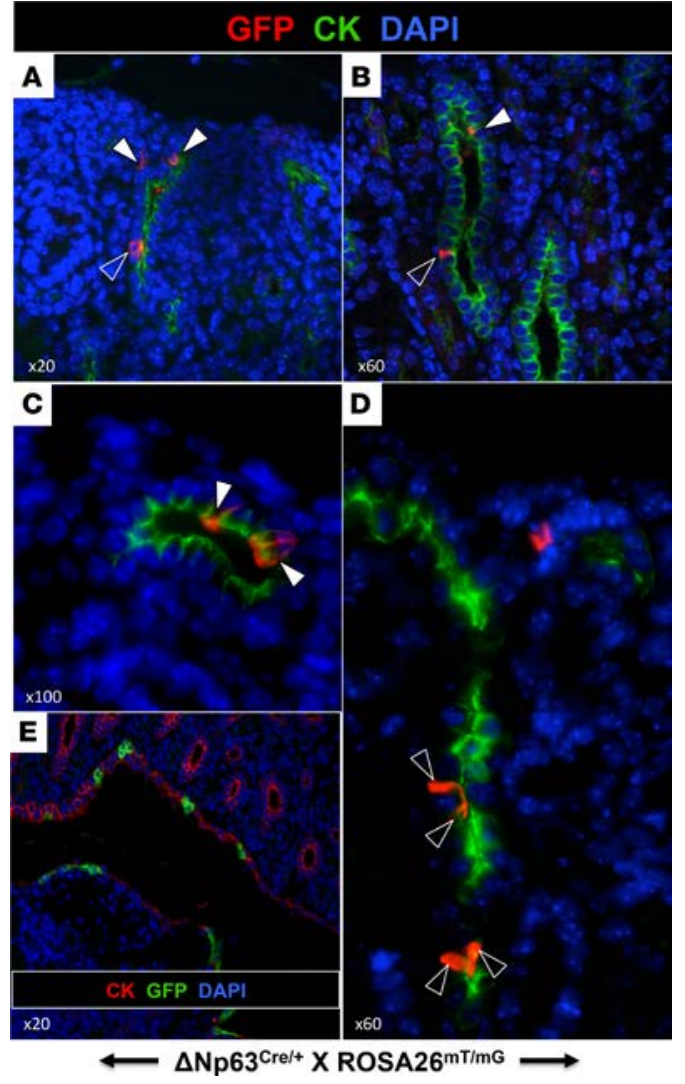

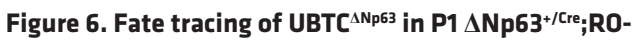
SA26 ${ }^{\mathrm{mT} / \mathrm{mC}}$ mice. Section immunofluorescence staining of the ureteric bud/collecting duct marker with cytokeratin (CK, green) and membrane-tethered GFP (red). Progeny of GFP+ UBTC ${ }^{\triangle N p 63}$ pattern the cortical collecting ducts and are found in both tips (A and $\mathbf{C}$; white arrowheads) and stalks (B and $\mathbf{D}$, outlined arrowheads). (E) Low-power image of the deep medullary region showing the presence of GFP+ UBTC ${ }^{\triangle N p 63}$ in the urothelium but not in adjacent medullary/papillary collecting ducts $(n=4)$. Original magnification, $\times 20$ (A and E); $\times 60(B$ and $\mathbf{D}) ; \times 100$ (C).

acid-secreting cells and renal tubular acidosis.

Although this study was not designed to address the mechanisms whereby $\Delta \mathrm{Np} 63$ programs the UBTC-to-IC fate, several ideas come to mind. $\Delta \mathrm{Np} 63$ may be required for the renewal or survival of a select subset of UBTC destined to give rise to IC; however, this hypothesis is countered by the integrity of $\mathrm{GFP}^{+}$tip cells, which remains intact in knockin $\Delta \mathrm{Np} 63^{\mathrm{gfp} / \mathrm{gfp}}$ mice. Another possibility is that $\Delta \mathrm{Np} 63$ acts upstream of Foxi1, a key transcriptional regulator of IC genes (32). A ChIP-seq screen of p63 target genes in neonatal kidney cortex did not reveal binding of p63 to the Foxil gene (Saifudeen and El-Dahr et al., unpublished observations), suggesting that Foxi1 is not a direct p63 target gene. We did, however, find binding of p63 to multiple genes in the Notch pathway, including Notch1, Notch2, Notch4, MAML3, Hes7, DLL1, Jag1,2 and RFNG. A previous study demonstrated binding of $\Delta \mathrm{Np} 63$ to the Notch3 gene in epidermal cells (26). It is tempting to speculate that $\Delta \mathrm{Np} 63$ promotes the IC fate indirectly by repressing the Notch pathway. It is also conceivable that $\Delta \mathrm{Np} 63$ "bookmarks" IC-specific genes, facilitating binding and activation by Foxi1. In this regard, $\triangle \mathrm{Np} 63$ has been shown to recruit chromatin remodelers to dynamic enhancers of epidermis-specific differentiation genes (35).

The coexpression of p63 in Ret $^{+}$tip progenitors prompts the question of whether $\mathrm{p} 63$ is a genomic target of Ret signaling. Review of public databases and the published UBTC transcriptome do not support this hypothesis. On the other hand, there is evidence that p63, jointly with Sox2, regulates the Ret target ETV4 gene (36). Since p63 expression is ini-

Figure 7. UBTC ${ }^{\mathrm{ANp63}}$ are precursors of cortical intercalated cells in the adult kidney. (A-D) $\triangle N p 63^{+/ / \text {re }}$ knockin mice were crossed to ROSA26 EYFP reporter

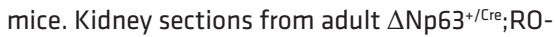
SA26 ${ }^{\mathrm{EYFP}}$ mice were immunostained to label $\triangle \mathrm{Np} 63$ daughter cells (EYFP), PC (AQP2), IC (vH-ATPase B1), A-type IC (AE1), and non-A-type IC (pendrin). EYFP+ cells stain positive for IC but not PC markers. (E) Model for ureteric bud tip cell (UBTC) differentiation UBTC differentiate into ureteric bud (UB) precursors of $\mathrm{PC}$ and IC; $\triangle \mathrm{Np} 63^{+}$cells located in UB tips differentiate to type $B-I C$, which in turn differentiate into type-A IC $(n=4)$. Original magnification, $\times 60$.

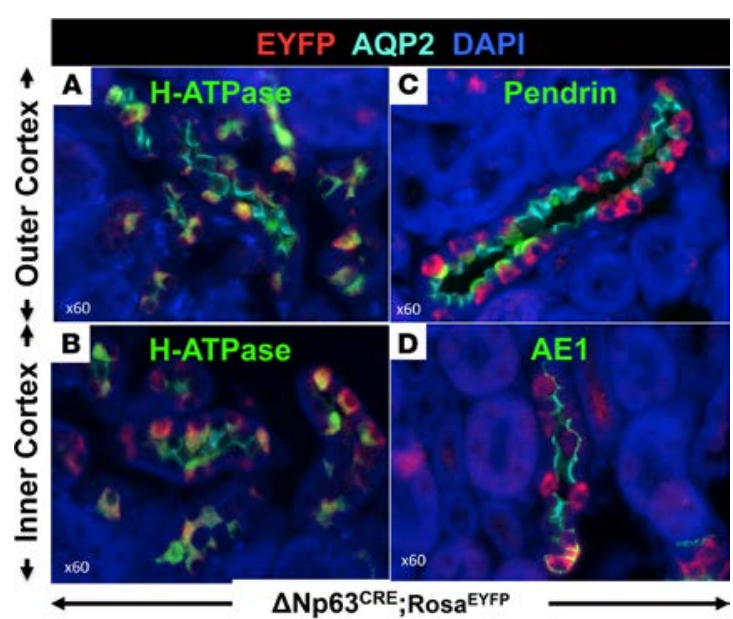

E

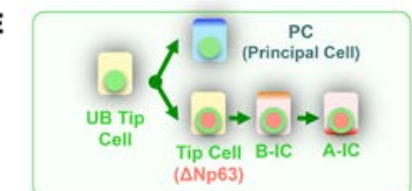




\section{EYFP AQP2 H-ATPase DAPI}

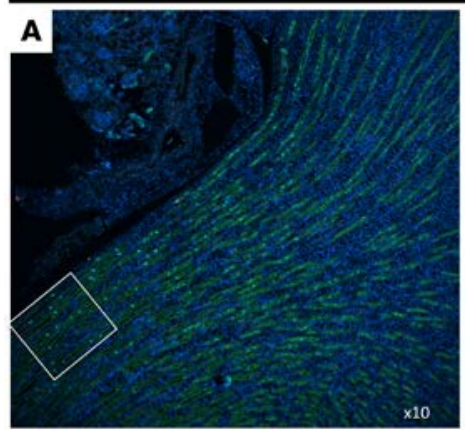

B

$\triangle$ Np63 ${ }^{\text {CRE }} ;$ ROSa $^{\text {EYFP }}$

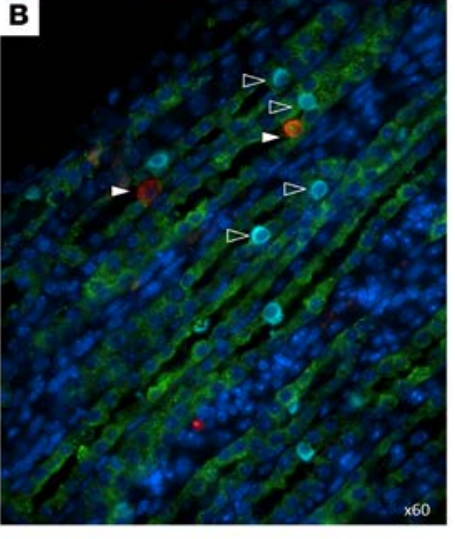

Figure 8. Occasional EYFP+ UBTC ${ }^{\text {ANp63lineage }}$ are seen in the medulla of adult kidney. However, these cells (red, white arrowheads) do not overlap with cells expressing the IC marker H-ATPase (blue, outlined arrowheads) $(n=4)$. Original magnification, $\times 10($ A); $\times 60$ (B).

tiated later than Ret in the developing kidney, it is still possible that GDNF/Ret or other tyrosine kinases may be upstream of p63. Future studies using conditional Ret or ETV4 mutants are needed to address this question.

In summary, the present study suggests that UBTC progenitors are not homogenous; at least a subset is specified early on to differentiate into cortical IC under the influence of $\Delta \mathrm{Np} 63$. Future studies are needed to address how p63 programs the fate of UBTC progenitors and whether theses mechanisms can be exploited in regenerative medicine.

\section{Methods}

Mice. $\Delta \mathrm{Np} 63^{+/ \text {Cre }}$ knockin mice were generated in the Signoretti laboratory (ref. 19) and were used to drive recombinase activity in $\Delta \mathrm{Np} 63$-expressing cells. Genotyping of these mice was performed as described

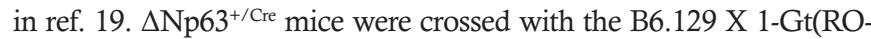
SA)26Sor ${ }^{\text {rml(EYFP)Cos } / J ~(R O S A 26 ~}{ }^{\text {EYFP) }}$ or B6.129(Cg)-Gt(ROSA)26Sor ${ }^{\text {T- }}$ m4(AcBB-ddTomato-EGFP)Luo/J (ROSA26 $6^{\text {mTomato/mEGFP) }}$ ) reporter mice from the Jackson Laboratories. Ret ${ }^{+/ E G F P}$ mice were provided by Sanjay Jain (Washington University, St. Louis, Missouri, USA).

Tissue preparation. Fetal and newborn mouse kidneys were fixed in $10 \%$ (vol/vol) buffered formalin at room temperature for 24 to 48 hours. Adult mice were euthanized and transcardially perfused with PBS, followed by $10 \%$ formalin. Adult organs were further fixed for 48 hours in formalin, before processing and embedding in paraffin.

Immunostaining. Immunohistochemistry and immunofluorescence were performed as described previously $(19,37)$. The following antibodies were used: mouse anti-pan-p63 (clone 4A4, Santa Cruz; clone SFI-6, Sigma-Aldrich; and DCS, Innovative Diagnostik-Systeme), rabbit anti- $\triangle \mathrm{Np63}$ (26), mouse anti-CK (Sigma-Aldrich, catalog C2562, 1:200), rabbit anti-Pax2 (Invitrogen, catalog 71-6000, 1:200), chicken anti-

Figure 9. Homozygous deletion of $\triangle \mathrm{Np} 63$ compromises IC differentiation. (A and B) H\&E staining showing intact overall structure of the nephrogenic zone in knockin $\Delta N p 63^{\text {fp/gfp }}$ mice. (C and D) Double immunostaining with p63 (red) and Pax2 (purple) showing that loss of $\triangle N p 63$ diminishes expression of total $p 63$ in UB tips. The insets show that NCAM (green) marks the induced cap mesenchyme and GFP labels $\triangle N p 63$ gene-targeted ureteric bud tip cells (UBTC). Loss of $\triangle N p 63$ has no effect on integrity of UBTC. ( $E$ and $\mathbf{F}$ ) Double immunostaining of AQP2 (red) and carbonic anhydrase II (CAII, green) showing that targeted deletion of $\triangle \mathrm{Np} 63$ results in loss of IC marker expression ( $n=$ 3 litters). Original magnification, $\times 20 ; \times 40$ (all insets).

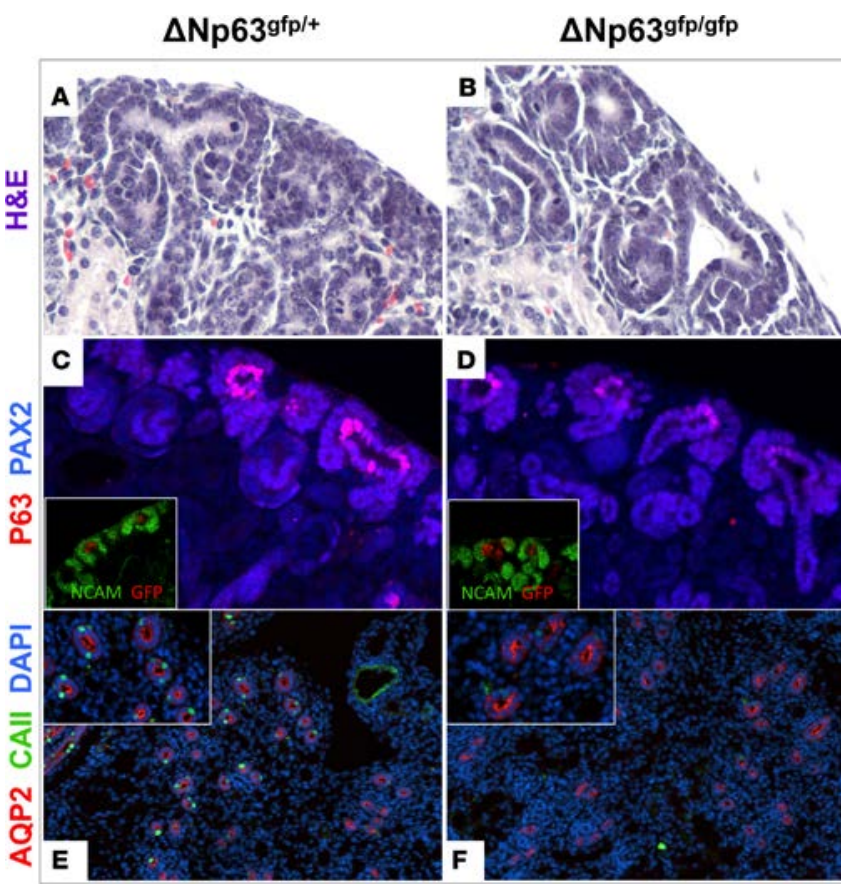


GFP (Abcam, catalog ab13970, 1:1000), mouse anti-E-cadherin (BD Biosciences, catalog 610181), rabbit v-H-ATPaseB1 (gift from Dennis Brown, Harvard Medical School, 1:1,000), rabbit anti-pendrin (gift from Susan Wall, Emory University, Atlanta, Georgia, USA; 1:20,000), rabbit anti-AE1 (Santa Cruz, 1:200), goat anti-AQP2 (Santa Cruz, 1:200), and goat anti-carbonic anhydrase 2 (Santa Cruz, 1:400). For immunofluorescence, image acquisition was performed using the Nikon Upright Microscope (Eclipse NI-U) and Nikon NIS-Elements Imaging Software (v4.40.00, build 1084, 64bit).

Study approval. Animal care and experiments were performed according to the guidelines of Tulane University School of Medicine for Animal Care Committee (under approved animal protocol 4341).

\section{Author contributions}

SSED and ZS conceived and designed the experiments. YL, JL, JCP, KSHS, and EG performed the experiments. SSED wrote the paper. S Signoretti and S Sinha contributed reagents and mice and revised the paper.

\section{Acknowledgments}

This work was supported by National of Institutes of Health grant RO1 DK62250 (to SSED). ZS is supported by National of Institutes of Health grant R56DK104779. We acknowledge the molecular core facility of the Tulane Renal and Hypertension Center, supported by the National Institute Of General Medical Sciences of the National Institutes of Health under award P30GM103337.

Address correspondence to: Samir S. El-Dahr or Zubaida Saifudeen, Tulane University School of Medicine, Department of Pediatrics, 1430 Tulane Avenue, New Orleans, Louisiana 70112, USA. Phone: 504.988.6692; E-mail: sedahr@tulane.edu (S.S. El-Dahr); zubisaif@tulane.edu (Z. Saifudeen).

This work was presented in part at the American Society of Nephrology meeting in San Diego, California, USA, on November 5-8, 2015.

1. Costantini F, Kopan R. Patterning a complex organ: branching morphogenesis and nephron segmentation in kidney development. Dev Cell. 2010;18(5):698-712.

2. Al-Awqati Q. Cell biology of the intercalated cell in the kidney. FEBS Lett. 2013;587(13):1911-1914.

3. Davis TK, Hoshi M, Jain S. To bud or not to bud: the RET perspective in CAKUT. Pediatr Nephrol. 2014;29(4):597-608.

4. Costantini F. Genetic controls and cellular behaviors in branching morphogenesis of the renal collecting system. Wiley Interdiscip Rev Dev Biol. 2012;1(5):693-713.

5. Packard A, et al. Luminal mitosis drives epithelial cell dispersal within the branching ureteric bud. Dev Cell. 2013;27(3):319-330.

6. Leclerc K, Costantini F. Mosaic analysis of cell rearrangements during ureteric bud branching in dissociated/reaggregated kidney cultures and in vivo. Dev Dyn. 2016;245(4):483-496.

7. Schmidt-Ott KM, et al. Novel regulators of kidney development from the tips of the ureteric bud. J Am Soc Nephrol. 2005;16(7):1993-2002.

8. Al-Awqati Q, Schwartz GJ. A fork in the road of cell differentiation in the kidney tubule. J Clin Invest. 2004;113(11):1528-1530.

9. Sampogna RV, Al-Awqati Q. Salt and pepper distribution of cell types in the collecting duct. J Am Soc Nephrol. 2013;24(2):163-165.

10. Kaelin WG. The emerging p53 gene family. J Natl Cancer Inst. 1999;91(7):594-598.

11. Moll UM, Erster S, Zaika A. p53, p63 and p73--solos, alliances and feuds among family members. Biochim Biophys Acta. 2001;1552(2):47-59.

12. Allocati N, Di Ilio C, De Laurenzi V. p63/p73 in the control of cell cycle and cell death. Exp Cell Res. 2012;318(11):1285-1290

13. Botchkarev VA, Flores ER. p53/p63/p73 in the epidermis in health and disease. Cold Spring Harb Perspect Med. 2014;4(8):a015248.

14. Melino G, Memmi EM, Pelicci PG, Bernassola F. Maintaining epithelial stemness with p63. Sci Signal. 2015;8(387):re9.

15. Mills AA, Zheng B, Wang XJ, Vogel H, Roop DR, Bradley A. p63 is a p53 homologue required for limb and epidermal morphogenesis. Nature. 1999;398(6729):708-713.

16. Signoretti S, et al. p63 is a prostate basal cell marker and is required for prostate development. Am J Pathol. 2000;157(6):1769-1775.

17. Koster MI, Kim S, Mills AA, DeMayo FJ, Roop DR. p63 is the molecular switch for initiation of an epithelial stratification program. Genes Dev. 2004;18(2):126-131.

18. Koster MI, Roop DR. p63 and epithelial appendage development. Differentiation. 2004;72(8):364-370.

19. Pignon JC, Grisanzio C, Geng Y, Song J, Shivdasani RA, Signoretti S. p63-expressing cells are the stem cells of developing prostate, bladder, and colorectal epithelia. Proc Natl Acad Sci U S A. 2013;110(20):8105-8110.

20. Zuo W, et al. p63(+)Krt5(+) distal airway stem cells are essential for lung regeneration. Nature. 2015;517(7536):616-620.

21. Schuchardt A, D'Agati V, Larsson-Blomberg L, Costantini F, Pachnis V. Defects in the kidney and enteric nervous system of mice lacking the tyrosine kinase receptor Ret. Nature. 1994;367(6461):380-383.

22. Jain S, Golden JP, Wozniak D, Pehek E, Johnson EM, Milbrandt J. RET is dispensable for maintenance of midbrain dopaminergic neurons in adult mice. J Neurosci. 2006;26(43):11230-11238.

23. Pellegrini G, et al. p63 identifies keratinocyte stem cells. Proc Natl Acad Sci U S A. 2001;98(6):3156-3161. 
24. Senoo M, Pinto F, Crum CP, McKeon F. p63 Is essential for the proliferative potential of stem cells in stratified epithelia. Cell. 2007;129(3):523-536.

25. Forster N, et al. Basal cell signaling by p63 controls luminal progenitor function and lactation via NRG1. Dev Cell. 2014;28(2):147-160

26. Romano RA, et al. $\Delta$ Np63 knockout mice reveal its indispensable role as a master regulator of epithelial development and differentiation. Development. 2012;139(4):772-782

27. Schwartz GJ, Barasch J, Al-Awqati Q. Plasticity of functional epithelial polarity. Nature. 1985;318(6044):368-371.

28. Takito J, Hikita C, Al-Awqati Q. Hensin, a new collecting duct protein involved in the in vitro plasticity of intercalated cell polarity. J Clin Invest. 1996;98(10):2324-2331.

29. Shakya R, Watanabe T, Costantini F. The role of GDNF/Ret signaling in ureteric bud cell fate and branching morphogenesis. Dev Cell. 2005;8(1):65-74.

30. Bridgewater D, et al. Canonical WNT/beta-catenin signaling is required for ureteric branching. Dev Biol. 2008;317(1):83-94

31. Marose TD, Merkel CE, McMahon AP, Carroll TJ. Beta-catenin is necessary to keep cells of ureteric bud/Wolffian duct epithelium in a precursor state. Dev Biol. 2008;314(1):112-126.

32. Vidarsson H, Westergren R, Heglind M, Blomqvist SR, Breton S, Enerbäck S. The forkhead transcription factor Foxi1 is a master regulator of vacuolar H-ATPase proton pump subunits in the inner ear, kidney and epididymis. PLoS One. 2009;4(2):e4471.

33. Blomqvist SR, et al. Distal renal tubular acidosis in mice that lack the forkhead transcription factor Foxi1. J Clin Invest. 2004;113(11):1560-1570.

34. Jeong HW, et al. Inactivation of Notch signaling in the renal collecting duct causes nephrogenic diabetes insipidus in mice. $J$ Clin Invest. 2009;119(11):3290-3300.

35. Kouwenhoven EN, et al. Transcription factor p63 bookmarks and regulates dynamic enhancers during epidermal differentiation. EMBO Rep. 2015;16(7):863-878.

36. Watanabe H, et al. SOX2 and p63 colocalize at genetic loci in squamous cell carcinomas. J Clin Invest. 2014;124(4):1636-1645

37. Chen S, Yao X, Li Y, Saifudeen Z, Bachvarov D, El-Dahr SS. Histone deacetylase 1 and 2 regulate Wnt and p53 pathways in the ureteric bud epithelium. Development. 2015;142(6):1180-1192. 